\title{
Anestesia por isofluorano em eqüinos submetidos à infusão contínua de medetomidina ou xilazina
}

\author{
Isoflurane anesthesia in horses during medetomidine or xilazine continuous infusion
Renata Gebara Sampaio Dória ${ }^{\mathrm{I}}$ Carlos Augusto Araújo ValadãoII Paulo Aléscio Canola Érica Cristina Bueno do Prado Guirro ${ }^{\mathrm{I}}$ Marina Ceccato Mendes $^{\mathrm{I}}$ André Escobar $^{\mathrm{I}}$ Gesiane Ribeiro ${ }^{\mathrm{I}}$ Cláudio Côrrea Natalini ${ }^{\mathrm{II}}$

RESUMO

Avaliaram-se oito eqüinos sob anestesia geral inalatória com isofluorano (1CAM) e infusão contínua de xilazina $\left(0,35 \mathrm{mg} \mathrm{kg}^{-1} \mathrm{~h}^{-1}\right)$ ou medetomidina $\left(3,5 \mu \mathrm{g} \mathrm{kg}^{-1} \mathrm{~h}^{-1}\right)$, em relação à freqüência cardíaca, ritmo cardiaco, freqüência respiratória, pressão arterial, hemogasometria arterial e temperatura, nos tempos T0 (imediatamente antes do início da infusão contínua) e T10 ao T60 (intervalos de 10 minutos, após inicio da infusão contínua). Houve redução da freqüência cardíaca e da temperatura e elevação da pressão arterial média. A paCO (no GM) elevou-se e a paO, mostrou-se maior no GM que no GX. Conclui-se que a infusão contínua de doses equipotentes de xilazina e medetomidina, durante anestesia geral inalatória, com isofluorano, em eqüinos, promove alterações cardiocirculatórias, respiratórias, térmicas e hemogasométricas discretas e equivalentes.

Palavras-chave: eqüinos, infusão contínua, isofluorano, medetomidina, xilazina.

\section{ABSTRACT}

Eight horses under inhalant general anesthesia with isoflurane (1MAC) and continuous infusion of xylazine $\left(0.35 \mathrm{mg} \mathrm{kg}^{-1} \mathrm{~h}^{-1}\right)$ or medetomidine $\left(3.5 \mu \mathrm{g} \mathrm{kg}^{-1} \mathrm{~h}^{-1}\right)$ were evaluated for heart rate and rhythm, respiratory rate, arterial blood pressure, arterial blood gas analysis and temperature immediately before the beginning of the continuous infusion (T0) and in intervals of 10 minutes after the beginning of the continuous infusion (T10 to T60). Heart rate and temperature decreased and mean arterial pressure increased. $\mathrm{PaCO}_{2}$ (in GM) increased and GM showed a higher paO, than $G X$. We conclude that equipotent doses of continuous infusion of medetomidine and xylazine during inhalant general anesthesia with isoflurane in horses promote slight and equivalent cardiocirculatory, respiratory, thermic and arterial blood gases changes.

Key words: medetomidine, xylazine, continuous infusion, isoflurane, equine.

\section{INTRODUÇÃO}

Atualmente, sabe-se que a elevada taxa de mortalidade, durante a anestesia, em eqüinos, tem como causas contribuitivas a depressão da função cardiovascular, hipotensão, redução do débito cardíaco, depressão respiratória, hipoventilação e hipercapnia associadas ao uso de agentes anestésicos voláteis, como o isofluorano, dependendo da dose e do tempo de exposição ao fármaco (STEFFEY \& HOWLAND, 1980; STEFFEY et al., 1987; STEFFEY, 1991; KUUSELA et al., 2001). Além disso, cavalos posicionados em decúbito dorsal sofrem depressão cardiovascular mais severa do que aqueles posicionados em decúbito lateral (BETTSCHARTWOLFENSBERGER etal., 2001a; KUSHIRO etal., 2005). Dessa forma, torna-se preferível minimizar a quantidade do agente inalatório utilizado, promovendo-se analgesia, associando-o a outros fármacos (EWING et al., 1993; BETTSCHART-WOLFENSBERGER et al., 2001a; MUIR, 2004; KUSHIRO et al., 2005).

IPrograma de Pós-graduação em Cirurgia Veterinária, Faculdade de Ciências Agrárias e Veterinárias (FCAV), Universidade Estadual Paulista (UNESP), Campus de Jaboticabal, Jaboticabal, SP, Brasil. Endereço para correspondência: Avenida Antártica, 788, casa 26. Residencial Villas Boas. Ribeirão da Ponte, 78040-500, Cuiabá, MT, Brasil. E-mail: renata-doria@uol.com.br. IIDepartamento de Clínica e Cirurgia Veterinária, FCAV, UNESP, Campus de Jaboticabal, Jaboticabal, SP, Brasil.

IIIDepartamento de Farmacologia, Universidade Federal do Rio Grande do Sul (UFRGS), Porto Alegre, RS, Brasil. 
A xilazina e a medetomidina são substâncias com propriedades sedativa e analgésica, altamente lipofílicas, com volume de distribuição alto, cuja meia vida de eliminação situa-se entre 30 a 90 minutos e de depuração total entre 20 e $80 \mathrm{~mL} \mathrm{~kg}^{-1} \mathrm{~min}^{-1}$, classificadas farmacologicamente como agonistas dos receptores adrenérgicos do tipo $\alpha_{2}$ (agonista $\left.\alpha_{2}\right)$ (SALONEN, 1992). A medetomidina, embora similar à xilazina, produz efeitos comportamentais mais potentes (WAGNER et al., 1991; BRYANT et al., 1996). Sugere-se que, embora possua um perfil farmacodinâmico similar aos outros agonistas $\alpha_{2}$ empregados em eqüinos, a medetomidina promova maior analgesia, sedação e ataxia que a xilazina devido, possivelmente, à penetração mais rápida da medetomidina no sistema nervoso central (SNC) (KAMERLING, et al., 1991; WAGNER et al., 1991; BRYANT et al., 1996; THURMON et al., 1996; BUENO et al., 1999), sendo as doses sedativas equipotentes para administração intravenosa de xilazina e medetomidina de $1 \mathrm{mg} \mathrm{kg} \mathrm{kg}^{-1}$ e 5 a $10 \mu \mathrm{g} \mathrm{kg}^{-1}$, respectivamente (YAMASHITA et al., 2000a; YAMASHITA et al., 2000b). A medetomidina tem mostrado ser segura para ser utilizada em esquemas de infusão contínua em eqüinos, uma vez que, em taxa de infusão adequada, produz sedação prolongada relacionada à manutenção de concentração plasmática estável. Na velocidade de infusão de $3,5 \mu \mathrm{g} \mathrm{kg}^{-1} \mathrm{~h}^{-1}$, promove um efeito sedativo constante, analgesia e mínimos efeitos cardiopulmonares (BETTSCHARTWOLFENSBERGER et al.,1999; 2001a).

O objetivo neste trabalho foi avaliar comparativamente, os efeitos cardiovasculares, hemogasométricos, respiratórios e variações de temperatura induzidas pela infusão contínua de medetomidina ou xilazina associadas à anestesia geral, inalatória com isofluorano, em eqüinos.

\section{MATERIAL E MÉTODOS}

Foram utilizados oito eqüinos, adultos, sem raça definida, considerados hígidos, após exames clínicos e laboratoriais, com peso corpóreo médio de $400 \pm 26 \mathrm{~kg}$, distribuídos em dois grupos experimentais: grupo xilazina (GX), representado pelos oito animais que receberam infusão contínua de xilazina e grupo medetomidina (GM), correspondendo aos oito animais que receberam infusão contínua de medetomidina. Os mesmos animais participaram dos dois grupos experimentais, respeitando-se um intervalo de pelo menos um mês entre cada procedimento.

Os animais foram submetidos a jejum alimentar de doze horas antes do início do experimento. O GX recebeu medicação pré-anestésica com midazolam $^{\mathrm{a}}\left(0,1 \mathrm{mg} \mathrm{kg}^{-1}\right)$, via intravenosa (IV) e xilazina ${ }^{\mathrm{b}}$ $\left(0,75 \mathrm{mg} \mathrm{kg}^{-1} \mathrm{IV}\right)$ e o GM recebeu midazolam $\left(0,1 \mathrm{mg} \mathrm{kg}^{-1}\right.$ IV) e medetomidina ${ }^{\mathrm{c}}\left(7,5 \mu \mathrm{g} \mathrm{kg}^{-1} \mathrm{IV}\right)$, ambos associados, com administração padronizada em um minuto. Aguardaram-se, então, 10 minutos para os animais serem submetidos à indução anestésica com cetamina $\mathrm{S}(+)\left(1 \mathrm{mg} \mathrm{kg}^{-1} \mathrm{IV}\right)$ cuja administração foi padronizada em 30 segundos. Ato contínuo, os eqüinos foram intubados com sonda de Magill ${ }^{\text {d }}$ compatível com o diâmetro da traquéia, posicionados em decúbito dorsal, na mesa cirúrgica, administrando-se-lhes oxigênio a $100 \%$, com fluxo de $20 \mathrm{~mL} \mathrm{~kg}^{-1}$ minuto ${ }^{-1}$, durante os 10 minutos iniciais, seguido de $10 \mathrm{~mL} \mathrm{~kg}^{-1}$ minuto ${ }^{-1}$, por meio de circuito anestésico semi-fechado ${ }^{\mathrm{e}}$, dotado de vaporizador calibrado para isofluorano ${ }^{\mathrm{f}}$.

Para o fornecimento do anestésico inalatório, a concentração foi ajustada em uma concentração alveolar mínima (1 CAM e 1,3\% de isofluorano), medida ao final da expiração, por analisador de gases anestésicos ${ }^{\mathrm{g}}$. Essas determinações das concentrações de isofluorano, inspiradas e expiradas, foram obtidas continuamente, por meio de colheitas de gases, realizadas através de tubo siliconado, fixado na parede interna da sonda traqueal cuja extremidade distal estava posicionada próxima à carina. Um filtro com $40 \mathrm{~g}$ de carvão ativado, substituído a cada anestesia, foi conectado, de forma intermitente, entre a extremidade proximal deste tubo fixado à sonda traqueal e o sensor do analisador de gases anestésicos, para mensurar a concentração de metano presente na amostra de gases expirados, evitando sua interferência na leitura do gás anestésico. Após a instrumentação dos animais e estabilização em 1 CAM de isofluorano (período padronizado em 10 minutos), o GX recebeu, por infusão contínua $^{\mathrm{h}}, 0,35 \mathrm{mg} \mathrm{kg}^{-1} \mathrm{~h}^{-1}$ de xilazina, diluída em solução físiológica (diluição 1:2) e o GM $3,5 \mu \mathrm{g} \mathrm{kg}^{-1} \mathrm{~h}^{-1}$ de medetomidina, diluída em solução fisiológica (diluição 1:10), durante o período de uma hora de avaliação.

A freqüência cardíaca e o ritmo cardíaco foram avaliados pelo traçado eletrocardiográfico, registrado por meio de eletrocardiografia computadorizada $^{j}$, na derivação base-ápice. A freqüência respiratória foi avaliada pela movimentação do gradil-costal. Utilizou-se um catéter, introduzido na artéria carótida esquerda, que fora previamente deslocada para o espaço subcutâneo (TAVERNOR, 1969), para mensurar a pressão arterial média (PAM), obtida pela leitura direta, do valor transduzido da onda de pulso mostrada no visor do monitor multiparamétrico ${ }^{1}$. Foi realizada hemogasometria arterial (pH arterial, pressão parcial de oxigênio, pressão parcial de gás carbônico, concentração de bicarbonato no plasma e excesso ou deficit de bases do sangue), por 
meio de amostras de sangue arterial, colhidas através do cateter e depositadas em receptáculo do analisador automático dos gases sanguíneos ${ }^{\mathrm{m}}$. A temperatura retal foi avaliada com um termômetro clínico convencional.

Os parâmetros mencionados foram avaliados nos seguintes tempos: T0 - avaliado após instrumentação dos animais e estabilização da profundidade anestésica em 1 CAM de isofluorano (padronizado 10 minutos após início da anestesia geral inalatória), imediatamente antes do início da infusão contínua; T10 - T60 - avaliados em intervalos de 10 minutos, após iniciar a infusão contínua dos fármacos, sendo então o período de avaliações de 60 minutos; após a última avaliação, os animais permaneceram 10 minutos sob anestesia geral inalatória com 1 CAM de isofluorano, tempo padronizado para retirada dos equipamentos e preparação dos animais para encaminhamento à sala de recuperação anestésica.

Os dados, obtidos na fase experimental, foram submetidos à análise de variância, para repetições múltiplas (RM ANOVA), para os dados paramétricos, seguida pelo teste de Student-Newman-Keuls (SNK). Entre os grupos, nos diferentes intervalos, foi realizada pelo teste-t de Student. As diferenças foram consideradas estatisticamente significativas, quando $\mathrm{P} \leq 0,05$.

\section{RESULTADOS}

Foi observada, no GX e no GM, redução significativa das médias de freqüência cardíaca, do T10 ao T60, em relação ao T0 (Tabela 1). Foi observada, no GX e no GM, elevação significativa das médias de pressão arterial média, do T40 ao T60, e nos T50 e T60, respectivamente, em relação ao T0 (Tabela 1). Foi observada, no GX e no GM, redução significativa das médias de temperatura, do T30 ao T60, em relação ao T0. Entre os grupos, foram observadas diferenças significativas entre T10 e T60, exceto T30, em que a temperatura do GM mostrou-se superior à do GX (Tabela 1). Foi observada, no GX, elevação significativa das médias de freqüência respiratória, do T10 ao T60, em relação ao T0 (Tabela 1). No GM, um animal apresentou apnéia, seguida de freqüência respiratória reduzida e amplitude elevada, durante os 10 minutos iniciais de anestesia inalatória,

Tabela 1 - Valores médios $\overline{\mathcal{X}}$ e desvios padrão $( \pm \mathrm{DP})$ da freqüência cardíaca $(\mathrm{FC} ; \mathrm{bpm})$, pressão arterial média $(\mathrm{PAM} ; \mathrm{mmHg})$, temperatura $\left({ }^{\circ} \mathrm{C}\right)$, freqüência respiratória $(f ; \mathrm{mpm}), \mathrm{pH}$ arterial, pressão parcial de oxigênio $\left(\mathrm{paO}_{2} ; \mathrm{mmHg}\right.$, pressão parcial de gás carbônico (paCO ${ }_{2}$; $\mathrm{mmHg})$, concentração de bicarbonato no plasma $\left(\mathrm{cHCO}_{3}^{-} ; \mathrm{mmol} \mathrm{L}^{-1}\right)$ e excesso ou deficit de bases do sangue $\left(\mathrm{EB} ; \mathrm{mmol} \mathrm{L}^{-1}\right)$, obtido em eqüinos anestesiados com isofluorano, sob infusão contínua de xilazina (GX) ou medetomidina (GM).

\begin{tabular}{|c|c|c|c|c|c|c|c|c|c|}
\hline & Tempo & & T0 & $\mathrm{T} 10$ & $\mathrm{~T} 20$ & $\mathrm{~T} 30$ & $\mathrm{~T} 40$ & $\mathrm{~T} 50$ & T60 \\
\hline \multirow{2}{*}{$\begin{array}{c}\text { FC } \\
(\mathrm{bpm})\end{array}$} & $\mathrm{GX}$ & $\overline{\mathrm{Y}}_{\mathrm{J}} \pm \mathrm{DP}$ & $52 \pm 7,9$ & $49 \pm 7,0^{\#}$ & $47 \pm 6,5^{\#}$ & $45 \pm 5,2^{\#}$ & $43 \pm 5,1^{\#}$ & $42 \pm 3,6^{\#}$ & $41 \pm 4,4^{\#}$ \\
\hline & GM & $\bar{\Upsilon}_{ \pm \mathrm{DP}}$ & $53 \pm 7,8$ & $49 \pm 8,5^{\#}$ & $49 \pm 7,4^{\#}$ & $48 \pm 6,5^{\#}$ & $47 \pm 6,0^{\#}$ & $45 \pm 4,9^{\#}$ & $44 \pm 4,7^{\#}$ \\
\hline \multirow{2}{*}{$\begin{array}{c}\text { PAM } \\
(\mathrm{mmHg})\end{array}$} & $\mathrm{GX}$ & $\overline{\bar{Q}}_{ \pm \mathrm{DP}}$ & $58 \pm 14$ & $65 \pm 17$ & $62 \pm 14$ & $68 \pm 13$ & $76 \pm 10^{\#}$ & $81 \pm 12^{\#}$ & $85 \pm 15^{\#}$ \\
\hline & GM & $\overline{\widehat{Q}}_{ \pm \mathrm{DP}}$ & $58 \pm 11$ & $58 \pm 10$ & $58 \pm 11$ & $62 \pm 14$ & $66 \pm 15$ & $71 \pm 14^{\#}$ & $74 \pm 14^{\#}$ \\
\hline \multirow{2}{*}{$\begin{array}{c}\mathrm{T} \\
\left({ }^{\circ} \mathrm{C}\right)\end{array}$} & GX & $\bar{Q}_{ \pm \mathrm{DP}}$ & $37,5 \pm 0,7$ & $37,2 \pm 0,6^{*}$ & $37,1 \pm 0,6^{*}$ & $37 \pm 0,8^{\#}$ & $36,9 \pm 0,8^{\# *}$ & $36,6 \pm 0,6^{\# *}$ & $36,7 \pm 0,6^{\# *}$ \\
\hline & GM & $\bar{\Upsilon}_{ \pm D P}$ & $37,8 \pm 0,4$ & $37,8 \pm 0,3^{*}$ & $37,7 \pm 0,3^{*}$ & $37,5 \pm 0,3^{\#}$ & $37,6 \pm 0,5^{\#} *$ & $37,4 \pm 0,3^{\#} *$ & $37,3 \pm 0,3^{\#} *$ \\
\hline \multirow{2}{*}{$\begin{array}{c}f \\
(\mathrm{mpm})\end{array}$} & GX & $\bar{\phi}_{ \pm} \pm \mathrm{DP}$ & $4 \pm 2,1$ & $5 \pm 1,4^{\#}$ & $5 \pm 1,3^{\#}$ & $5 \pm 1,5^{\#}$ & $5 \pm 1,5^{\#}$ & $5 \pm 1,3^{\#}$ & $5 \pm 1,4^{\#}$ \\
\hline & GM & $\bar{\varphi}_{\mathrm{JP}} \pm \mathrm{DP}$ & $5 \pm 2,0$ & $6 \pm 2,5$ & $5 \pm 2,3$ & $6 \pm 1,9$ & $6 \pm 2,2$ & $6 \pm 2,2$ & $6 \pm 2,0$ \\
\hline \multirow[t]{2}{*}{$\mathrm{pH}$} & GX & $\overline{\bar{X}} \pm \mathrm{DP}$ & $7,30 \pm 0,02$ & $7,33 \pm 0,02^{\#}$ & $7,33 \pm 0,01^{\#}$ & $7,33 \pm 0,01^{\#}$ & $7,32 \pm 0,02^{\#}$ & $7,32 \pm 0,02^{\#}$ & $7,33 \pm 0,02^{\#}$ \\
\hline & GM & $\bar{\Upsilon}_{\mathrm{J}} \pm \mathrm{DP}$ & $7,33 \pm 0,04$ & $7,33 \pm 0,04$ & $7,32 \pm 0,04$ & $7,32 \pm 0,03$ & $7,32 \pm 0,03$ & $7,33 \pm 0,04$ & $7,33 \pm 0,04$ \\
\hline \multirow{2}{*}{$\begin{array}{c}\mathrm{paO}_{2} \\
(\mathrm{mmHg})\end{array}$} & GX & $\bar{Q}_{1} \pm \mathrm{DP}$ & $161 \pm 56$ & $168 \pm 41 *$ & $180 \pm 58$ & $177 \pm 62$ & $142 \pm 27 *$ & $134 \pm 25^{*}$ & $129 \pm 32 *$ \\
\hline & GM & $\overline{\mathrm{Y}}_{ \pm \mathrm{DP}}$ & $223 \pm 67$ & $227 \pm 57^{*}$ & $216 \pm 58$ & $213 \pm 54$ & $208 \pm 70 *$ & $210 \pm 80^{*}$ & $203 \pm 83 *$ \\
\hline \multirow{2}{*}{$\begin{array}{c}\mathrm{paCO}_{2} \\
(\mathrm{mmHg})\end{array}$} & GX & $\bar{\Upsilon}_{ \pm D P}$ & $57,0 \pm 6$ & $54,8 \pm 2$ & $56,2 \pm 2$ & $58,5 \pm 7$ & $57,0 \pm 8$ & $59,6 \pm 5$ & $58,9 \pm 6$ \\
\hline & GM & $\bar{P}_{ \pm D P}$ & $54,5 \pm 5$ & $58,2 \pm 6^{\#}$ & $61,3 \pm 9^{\#}$ & $59,7 \pm 5^{\#}$ & $59,9 \pm 1^{\#}$ & $62,2^{\#} \pm 9$ & $63,6^{\#} \pm 8$ \\
\hline \multirow{2}{*}{$\begin{array}{c}\mathrm{cHCO}_{3}^{-} \\
\left(\mathrm{mmol} \mathrm{L}^{-1}\right)\end{array}$} & GX & $\bar{\phi}_{ \pm \mathrm{DP}}$ & $27,4 \pm 1,8$ & $28,0 \pm 1,3$ & $28,7 \pm 1,7$ & $28,8 \pm 1,6$ & $28,8 \pm 3,6$ & $30,4 \pm 1,7^{\#}$ & $30,3 \pm 1,8^{\#}$ \\
\hline & GM & $\bar{\Upsilon}_{ \pm D P}$ & $27,7 \pm 1,5$ & $29,4 \pm 2,0^{\#}$ & $30,1 \pm 2,3^{\#}$ & $30,0 \pm 2,1^{\#}$ & $29,9 \pm 4,7^{\#}$ & $31,3 \pm 2,0^{\#}$ & $32,1 \pm 2,3^{\#}$ \\
\hline \multirow{2}{*}{$\begin{array}{c}\text { EB } \\
\left(\mathrm{mmol} \mathrm{L}^{-1}\right)\end{array}$} & GX & $\bar{\varphi}_{ \pm \mathrm{DP}}$ & $0,4 \pm 1,5$ & $1,4 \pm 1,1^{\#}$ & $2,0 \pm 1,6^{\#}$ & $2,1 \pm 1,4^{\#}$ & $2,1 \pm 3,0^{\#}$ & $3,4 \pm 1,4^{\#}$ & $3,4 \pm 1,5^{\#}$ \\
\hline & GM & $\bar{\varphi}_{ \pm \mathrm{DP}}$ & $1,3 \pm 1,4$ & $2,8 \pm 1,7^{\#}$ & $3,2 \pm 1,8^{\#}$ & $3,1 \pm 1,9^{\#}$ & $2,9 \pm 4,1^{\#}$ & $4,4 \pm 1,5^{\#}$ & $5,1 \pm 1,8^{\#}$ \\
\hline
\end{tabular}

\# diferença significativa, quando comparada a T0 (teste de Student-Newman-Keuls, $\mathrm{P}=0,05$ ).

* diferença significativa entre os grupos (teste $t$ de Student, $\mathrm{P}=0,05$ ). 
após a indução anestésica (período de instrumentação). Um animal apresentou apnéia por um período além dos 10 minutos iniciais, seguida por uma freqüência respiratória muito baixa, o que dificultou o estabelecimento de 1 CAM de isofluorano, nos tempos padronizados pelo experimento. Esse animal teve seus dados excluídos do experimento. Foi observada, no GX, elevação significativa das médias de pH arterial, do T10 ao T60, em relação ao T0 (Tabela 1). Em relação às médias de $\mathrm{paO}_{2}$, entre os grupos, foram observadas diferenças significativas nos T10, T40, T50 e T60, em que as paO, do GM mostraram-se superiores às do GX (Tabela 1 ). Foi observada, no $\mathrm{GM}$, elevação significativa das médias de $\mathrm{paCO}_{2}$, do T10 ao T60, em relação ao T0 (Tabela 1). Foi observada, no GX e no GM, elevação significativa das médias de $\mathrm{CHCO}_{3}^{-}$nos T50 e T60 e do T10 ao T60, respectivamente, em relação ao T0 (Tabela 1). Foi observada, no GX e no GM, elevação significativa das médias de EB, do T10 ao T60, em relação ao T0 (Tabela 1).

\section{DISCUSSÃO}

No tocante à avaliação cardiovascular dos eqüinos sob infusão contínua de agonistas $\alpha_{2}$, durante anestesia geral inalatória com isofluorano, neste estudo houve redução da freqüência cardíaca, embora os valores tenham permanecido dentro dos considerados fisiológicos (25 a 50bpm) (HUBELL, 2004). A pressão arterial média, durante a avaliação experimental, apresentou valores considerados, inicialmente, menores que o aceitável para uma anestesia geral, em eqüinos. Segundo HUBBELL (2004), a pressão arterial média deve manter-se entre 60 e $90 \mathrm{mmHg}$, em eqüinos anestesiados. Pressão arterial média menor que $60 \mathrm{mmHg}$ está associada à perda da autoregulação do fluxo sangüíneo no leito vascular e deve ser evitada. É importante porém, ressaltar que, apesar da ausência de estímulo cirúrgico neste estudo, após 10 minutos no GX e 30 minutos no GM, a PAM retornou a valores acima de $60 \mathrm{mmHg}$. Este fato, provavelmente, reflete a redução da concentração plasmática, após a redistribuição da dose inicial dos agonistas $\alpha_{2}$, sendo o efeito residual da medetomidina mais prolongado, o que corrobora com os estudos de YAMASHITA et al. (2000a), justificando, em parte, a maior duração da depressão cardiovascular produzida pela medetomidina em relação à xilazina. Da mesma forma, neste estudo, após 40 e 50 minutos do início da infusão contínua de xilazina e de medetomidina, respectivamente, os valores médios da pressão arterial média apresentaram-se, significativamente, maiores que os valores desta no momento anterior ao início da infusão contínua. Como se vê, pode-se suspeitar que uma redução nas doses dos agonistas $\alpha_{2}$ na sedação, provavelmente, reduziria essa depressão inicial na pressão arterial.

Em pôneis, BETTSCHARTWOLFENSBERGER et al. (1999) demonstraram que, durante duas horas de infusão contínua de $3,5 \mu \mathrm{g} \mathrm{kg}^{-1} \mathrm{~h}^{-1}$ de medetomidina, a freqüência cardíaca permaneceu similar aos valores de quando não sedados e a pressão arterial mostrou-se levemente reduzida, embora esses animais não estivessem submetidos à anestesia inalatória. No estudo ora finalizado, a discreta diminuição na freqüência cardíaca, resultou da bradicardia por diminuição do tônus simpático devido à redução na liberação de norepinefrina no locus coeruleus (BRYANT et al., 1996; BETTSCHARTWOLFENSBERGER et al., 2001b). A despeito disso, BETTSCHART-WOLFENSBERGER et al. (2001a) demonstaram que, em pôneis, a infusão contínua de $3,5 \mu \mathrm{g} \mathrm{kg}^{-1} \mathrm{~h}^{-1}$ de medetomidina, durante anestesia inalatória com desfluorano, não altera os parâmetros cardiovasculares. As alterações cardiovasculares induzidas pela administração em bolus seguida de infusão contínua de agonistas $\alpha_{2}$, como a xilazina e a medetomidina, comprovaram-se mínimas, assim como foi verificado em estudos que avaliavam a sedação promovida por esses fármacos (BRYANT et al, 1996) e, de acordo com este estudo e com BETTSCHARTWOLFENSBERGER et al. (2001a), um bolus de 7,5 $\mu \mathrm{g}$ $\mathrm{kg}^{-1}$ de medetomidina ou $0,75 \mathrm{mg} \mathrm{kg}^{-1}$ de xilazina, seguido da infusão contínua de $3,5 \mu \mathrm{g} \mathrm{kg}^{-1} \mathrm{~h}^{-1}$ de medetomidina ou $0,35 \mathrm{mg} \mathrm{kg}^{-1} \mathrm{~h}^{-1}$ de xilazina, pode ser considerado útil na anestesia de cavalos, produzindo analgesia, a despeito desses efeitos cardiovasculares discretos, em adição àqueles induzidos pelo agente anestésico inalatório.

Neste estudo, a administração do bolus de medetomidina alterou a freqüência respiratória e, em dois animais, produziu apnéia. Da mesma maneira, YAMASHITA et al. (2000a) relataram redução dosedependente da freqüência respiratória e $\mathrm{paO}_{2}$, após administração intravenosa de medetomidina, em eqüinos. Contudo, durante o período de anestesia geral inalatória e infusão contínua de medetomidina, não houve alteração da freqüência respiratória, sendo que esta permaneu dentro de valores considerados desejáveis para a anestesia inalatória, em eqüinos $(f$ entre 4 e 10mpm) (HUBELL, 2004). Por outro lado, o estudo de BETTSCHART-WOLFENSBERGER et al. (1999) relatou alguns casos de elevação da freqüência respiratória após 10 a 20 minutos do bolus de medetomidina em pôneis, e esta continuou até o final da infusão contínua. Similarmente, neste estudo, 
durante o período de infusão contínua de xilazina, houve elevação discreta da freqüência respiratória, embora dentro dos valores fisiológicos, provavelmente, um resultado da redução da concentração plasmática dos fármacos de tranqüilização e indução anestésica.

Os anestésicos inalatórios causam depressão cardiopulmonar de maneira dosedependente em eqüinos (STEFFEY, 1996). Segundo BETTSCHART-WOLFENSBERGER et al. (2001c), a medetomidina não causa acidose respiratória, durante a anestesia em eqüinos, por aumento na $\mathrm{paCO}_{2}$. $\mathrm{Da}$ mesma forma, neste estudo, não houve alteraçã̃o de $\mathrm{pH}$ no GM e, no GX, após um $\mathrm{pH}$ inicial ácido (provavelmente, devido aos efeitos da tranqüilização e indução anestésica), houve uma discreta elevação deste, durante todo o período da anestesia geral sob infusão contínua de xilazina, sendo que, nos dois grupos, os valores permaneceram, embora no limite inferior, dentro dos considerados fisiológicos $(\mathrm{pH}$ normal entre 7,32 e 7,44), mesmo após 60 minutos de anestesia (CARLSON, 1997). Os valores da $\mathrm{paCO}_{2}$, durante a anestesia geral inalatória e infusão contínua de medetomidina, apresentaram-se superiores aos desejáveis para uma anestesia em eqüinos $\left(\mathrm{paCO}_{2}=\right.$ $40 \mathrm{mmHg}$ ), caracterizando uma hipoventilação (MUIR et al., 2001), provavelmente, devido à depressão respiratória inicial, após a sedação e indução anestésica, além do decúbito dorsal associado à anestesia geral inalatória (YAMASHITA et al., 2000a). Isso também explica os valores de $\mathrm{paCO}_{2}$ acima de $40 \mathrm{mmHg}$, no grupo xilazina, durante todo o período anestésico, sob infusão contínua de xilazina e medetomidina. Conseqüentemente, ocorreu um aumento, no decorrer dessa anestesia, nos dois grupos experimentais, de bicarbonato e excesso de bases, além dos valores considerados fisiológicos $\left(\mathrm{HCO}_{3}^{->}>30 \mathrm{mmol}\right.$ $\mathrm{L}^{-1}$ e EB $\left.>3 \mathrm{mmol} \mathrm{L}^{-1}\right)$, caracterizando uma resposta metabólica compensatória ao excesso de $\mathrm{CO}_{2}$, mantendo, então, os valores de $\mathrm{pH}$ sangüíneo dentro da normalidade (MUIR et al., 2001). Neste estudo, a $\mathrm{paO}_{2}$ mostrou-se maior no GM que no GX, durante a anestesia geral, após início da infusão contínua, talvez, devido a uma maior hipertensão vascular pulmonar promovida pela xilazina. Aredução na taxa de ventilaçãoperfusão induzida pela provável diminuição do índice cardíaco e a vasoconstricção dos vasos pulmonares pode ter sido responsável por um decréscimo na $\mathrm{paO}_{2}$ (YAMASHITA et al., 2000a).

Neste estudo, houve redução da temperatura, após 30 minutos de anestesia geral inalatória e infusão contínua de xilazina ou medetomidina, mas de menos de $1^{\circ} \mathrm{C}$, o que é considerado aceitável. Muitos agonistas $\alpha_{2}$ afetam a termorregulação sendo este mecanismo dose e espécie-dependente (HALL \& CLARKE, 1991). É importante lembrar porém, vale lembrar que os animais deste experimento estavam sob anestesia geral inalatória, a qual também deprime a temperatura. Adicionalmente, a infusão contínua de medetomidina produziu menor redução de temperatura que a xilazina, durante todo o período experimental.

\section{CONCLUSÕES}

Com base nos resultados obtidos, pode-se concluir que a infusão contínua de $3,5 \mu \mathrm{g} \mathrm{kg}^{-1} \mathrm{~h}^{-1} \mathrm{de}$ medetomidina ou $0,35 \mathrm{mg} \mathrm{kg}^{-1} \mathrm{~h}^{-1}$ de xilazina promove depressão cardiocirculatória, respiratória, térmica e alterações hemogasométicas similares e discretas que não comprometem a segurança da anestesia geral inalatória, com isofluorano, em eqüinos.

\section{AGRADECIMENTO}

À Fundação de Amparo à Pesquisa do Estado de São Paulo (FAPESP), pela concessão da bolsa de estudo (processo $\mathrm{n}^{\circ} 04 / 12739-1$ ) e auxílio à pesquisa (processo $\mathrm{n}^{\circ} 2006 / 00645$ 8), que acreditou e apoiou este estudo experimental.

\section{FONTES DE AQUISIÇÃO}

a-Dormire - Cristália Produtos Químicos e Farmacêuticos Ltda., Itapira-SP, Brasil.

b-Coopazine 2\% - Schering-Plough, Cotia-SP, Brasil.

c-Domitor - Pfizer Animal Health, Inc, Extos, PA, EUA.

d-Sonda de Magill - Cirurg Fernandes Ltda, São Paulo-SP, Brasil. e-Aparelho de anestesia inalatória - HB Comercial Hospitalar Ltda, São Paulo-SP, Brasil.

f-Vaporizador calibrado para isofluorano - HB Comercial Hospitalar Ltda, São Paulo-SP, Brasil.

g-OHMEDA mod. 5250, processo FAPESP n ${ }^{\circ}$ 96/12830-0, EUA.

h-SAGE ATI orion - model 362, EUA.

i-Fisiológico (Cloreto de Sódio 0,9\%) - JP Indústria Farmacêutica S. A., Riberão Preto-SP, Brasil.

j-TEB - Mod. ECGPC software versão 1.10 - Processo FAPESP 96/1151-1.

1-Dixtal - mod. DX2010 - Módulo de Pressão Arterial Invasiva

- Manaus-AM, Brasil.

m-OMNI C Roche Diagnostics 2858, São Paulo, SP - Processo FAPESP 02/140540.

\section{COMISSÃO DE ÉTICA E BIOSSEGURANÇA}

Este estudo foi aprovado pela Comissão de Ética e do Bem-Estar dos Animais (CEBEA) do Campus de Jaboticabal da UNESP, conforme protocolo n ${ }^{\circ}$ 004499-05.

\section{REFERÊNCIAS}

BETTSCHART-WOLFENSBERGER, R. et al. Minimal alveolar concentration of desflurane in combination with an infusion of medetomidine for the anaesthesia of ponies. Veterinary 
Record, v.148, p.264-267, 2001a.

BETTSCHART-WOLFENSBERGER, R. et al. Cardiopulmonary effects of prolonged anesthesia via propofol-medetomidine infusion in ponies. American Journal of Veterinary Research, v.62, p.1428-1435, 2001 b.

BETTSCHART-WOLFENSBERGER, R. et al. Infusion of a combination of propofol and medetomine for long-term anesthesia in ponies. American Journal of Veterinary Research, v.62, p.500-507, 2001c.

BETTSCHART-WOLFENSBERGER, R. et al. Pharmacokinetics of medetomidine in ponies and elaboration of medetomidine infusion regime which provides a constant level of sedation. Research in Veterinary Science, v.67, p.41-46, 1999.

BRYANT, C.E. et al. Cardiopulmonary effects of medetomidine in sheep and in ponies. Research in Veterinary Science, v.60, p.267-271, 1996.

BUENO, A.C. et al. Cardiopulmonary and sedative effects of intravenous administration of low doses of medetomidine and xylazine to adult horses. American Journal of Veterinary Research, v.60, p.1371-1376, 1999.

CARLSON, G.P. Fluid, electrolyte and acid-base balance. In: KANEKO, J.J. et al. Clinical biochemistry of domestic animals. 5.ed. California: Academic, 1997. p.485-516.

EWING, K.K. et al. Reduction of isoflurane anesthetic requirement by medetomidine and its restoration by atipamezole in dogs. American Journal of Veterinary Research, v.54, p.294-299, 1993.

HALL, L.W.; CLARKE, K.W. Anaesthesia of the horse. In: Veterinary anaesthesia. 9.ed. London: Baillière $\overline{\text { Tindall, }}$ 1991. p.191-235.

HUBBELL, J.A.E. Anesthesia of the horse: monitoring, recovery, and complications. In: ANNUAL CONVENTION OF THE AMERICAN ASSOCIATION OF EQUINE PRACTITIONERS, 50., 2004, Denver, Colorado. Proceedings... Lexington: American Association of Equine Practitioners, 2004. Capturado em 09 jun. 2006. Online. Disponível em: http://www.ivis.org.

KAMERLING, S. et al. Pharmacological profile of medetomidine in the equine. Acta Veterinaria Scandinavica, v.87, suplemento, p.161-162, 1991.

KUSHIRO, T. et al. Anesthetic and cardiovascular effects of balanced anesthesia using constant rate infusion of midazolamketamine-medetomidine with inhalation of oxygen-sevoflurane (MKM-OS Anesthesia) in horses. Journal of Veterinary Medical Science, v.67, p.379-384, 2005.
KUUSELA, E. et al. Comparison of medetomidine and dexmedetomidine as premedicants in dogs undergoing propofolisoflurane anesthesia. American Journal of Veterinary Research, v.62, p.1073-1080, 2001.

MUIR, W.W. et al. Equilíbrio ácido-básico e gases sangüíneos. In: Manual de anestesia veterinária. 3.ed. Porto Alegre: Artmed, 2001. p.229-241.

MUIR, W.W. New perspectives on the drugs used to produce sedation, analgesia, and anesthesia in horses. In: ANNUAL CONVENTION OF THE AMERICAN ASSOCIATION OF EQUINE PRACTITIONERS, 50., 2004, Denver, Colorado. Proceedings... Lexington: American Association of Equine Practitioners, 2004. Capturado em 09 jun. 2006. Online. Disponível em: http://www.ivis.org.

SALONEN, J.S. Chemistry and pharmacokinetics of the $\alpha_{2}$ adrenoceptor agonistis. n: SHORT C. E.; POZNAK, A. Van. Animal pain. New York: Churchill Livingstone, 1992. p.191200 .

STEFFEY E. P.; HOWLAND D. Jr. Comparison of circulatory and respiratory effects of isoflurane and halotane anethesia in horses. American Journal of Veterinary Research, Chicago, v.41. p.821-825, 1980.

STEFFEY, E.P. et al. Cardiopulmonary function during 5 hours of constant-dose isoflurane in laterally recumbent, spontaneously breathing horses. Journal of Veterinary Pharmacology and Therapeutics, v.10, p.290-297, 1987.

STEFFEY, E.P. Inhalation anesthetics and gases. In: MUIR III, W.W.; HUBBELL, J.A.E. Equine anesthesia: monitoring and emergency therapy. St. Louis: MosbyYear Book, 1991. p.352-379.

STEFFEY, E.P. Inhalation anesthetics. In: THURMON, J.C. et al. Lumb \& Jones' veterinary anesthesia. 3.ed. Baltimore: Williams \& Wilkins, 1996. p.183-209.

TAVERNOR, W.D. Technique for the subcutaneous relocation of the common carotid artery in the horse. American Journal of Veterinary Research, v.30, p.1881-1883, 1969.

THURMON, J.C. et al. Preanesthetics and anesthetic adjuncts. In: _. Lumb \& Jones' veterinary anesthesia. 3.ed. Baltimore: Williams \& Wilkins, 1996 . p.183-209.

WAGNER, A.E. et al. Cardiovascular effects of xylazine and detomidine in horses. American Journal of Veterinary Research, v.52, p.651-657, 1991.

YAMASHITA, K. et al. Cardiovascular effects of medetomidine, detomidine and xylazine in horses. Journal of Veterinary Medical Science, v.62, n.10, p.1025-1032, 2000a.

YAMASHITA, K. et al. Combination of continous intravenous infusion using a mixture of guaifenesin-ketamine-medetomidine and sevoflurane anesthesia in horses. Journal of Veterinary Medical Science, v.62, p.229-235, 2000 b. 